\title{
Vanishing point detection for road detection
}

\author{
Hui Kong ${ }^{1,2,3} \quad$ Jean-Yves Audibert ${ }^{2,3} \quad$ Jean Ponce ${ }^{1,3}$ \\ ${ }^{1}$ École Normale Supérieure \\ Paris, France \\ ${ }^{2}$ Imagine, LIGM, Université Paris-Est \\ Paris, France \\ hui.kong@ens.fr audibert@imagine.enpc.fr jean.poncedens.fr
}

\begin{abstract}
Given a single image of an arbitrary road, that may not be well-paved, or have clearly delineated edges, or some a priori known color or texture distribution, is it possible for a computer to find this road? This paper addresses this question by decomposing the road detection process into two steps: the estimation of the vanishing point associated with the main (straight) part of the road, followed by the segmentation of the corresponding road area based on the detected vanishing point. The main technical contributions of the proposed approach are a novel adaptive soft voting scheme based on variable-sized voting region using confidence-weighted Gabor filters, which compute the dominant texture orientation at each pixel, and a new vanishingpoint-constrained edge detection technique for detecting road boundaries. The proposed method has been implemented, and experiments with 1003 general road images demonstrate that it is both computationally efficient and effective at detecting road regions in challenging conditions.
\end{abstract}

\section{Introduction}

Numerous image-based road (border) detection algorithms have emerged as one of the components of fully automatic vehicle navigation systems [10]. Most of the early systems have focused on following the well-paved structured road that is readily separated from its surroundings. More recently, triggered by the DARPA Grand Challenge [1], a competition between autonomous off-road vehicles in the Mojave desert, many algorithms have attempted to handle off-road conditions. Although significant advances have been made on specialized systems for detecting individual road types, little progress has been made in proposing a general algorithm to detect a variety of types of roads.

Given a road image as shown in Fig.1, can the computer

${ }^{3}$ WILLOW project-team, Laboratoire d'Informatique de l'École Normale Sup'erieure, ENS/INRIA/CNRS UMR 8548.

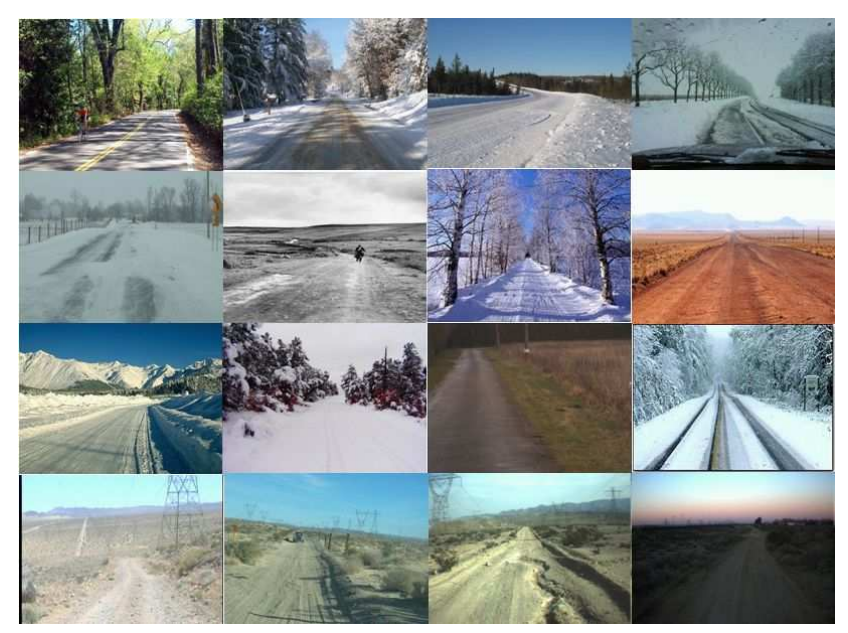

Figure 1. Different types of roads with varying colors, textures and lighting conditions.

roughly determine where the road is? This paper answers this question by proposing a novel framework for segmenting the road area based on the estimation of the vanishing point associated with the main (straight) part of the road. The novelties of this paper lie in the following aspects: (1) In the estimation of texture orientation, we not only compute the texture orientation at each pixel, but also give a confidence to each estimation. The introduced confidence is then incorporated into the vanishing point estimation. (2) Observing that the higher image pixels tend to receive more votes than lower image pixels, which usually results in wrong vanishing point estimation for the road images where the true vanishing point of the road is not in the upper part of the image, a locally adaptive soft-voting (LASV) scheme is proposed to overcome this problem. This vanishing point estimation method is much more efficient than previous texture-based methods. The scheme uses adaptivesized local voting region, in which pixels having low confidence texture orientation estimation are discarded. (3) To segment the road area, a vanishing-point constrained group of dominant edges are detected based on an Orientation Consistency Ratio (OCR), and from which two most domi- 
nant edges are selected as the road borders. Since the color cue is not used, this road detection method handles well changes of illumination and applies to general road images.

\section{Related work}

For structured roads, the localization of road borders or road markings is one of the most commonly used approach. Laser [15], radar [8], stereovision [3], color cue [16], Hough transform [14, 18], steerable filters [5], Spline model [17] etc. have been utilized to find the road boundaries or markings. The drawbacks of these methods is that they only consistently work for structured roads with noticeable markings or borders. Methods based on segmenting the road using the color cue have also been proposed but they do not work well for general road image, specially when the roads have little difference in colors between their surface and the environment.

For unstructured roads or structured roads without remarkable boundaries and markings, Alon et al. [2] have combined the Adaboost-based region segmentation and the boundary detection constrained by geometric projection to find the "drivable" road area. However, it needs many different types of road images to train a region classifier, which might be onerous. Reverse optical flow technique [7] provides an adaptive segmentation of the road area, but the method does not work well on chaotic roads when the camera is unstable and the estimation of the optical flow is not enough robust. Stereo camera $[4,9]$ are also used to determine terrain traversability. When there is little difference in color between the road surface and off-road area, it is hard to find strong intensity change to delimit them. The one characteristic that seems to define the road in such situations is texture. The associated approaches [11, 12, 13] have attempted to define the forward "drivable" image region by utilizing the texture cue. They rely on computing the texture orientation for each pixel, then seek the vanishing point of the road by a voting scheme, and finally localize the road boundary using the color cue. Our approach belongs to this line of research. Our main contributions are: a texture orientation estimation at each pixel of the image for which a confidence level is provided (Section 3), a voting scheme taking into account this confidence level and the distance from the voting pixel to the vanishing point candidate (Section 4), and a new vanishing-point constrained edge detection technique for finding the boundaries of the road (Section 5).

\section{Confidence-weighted texture orientation es- timation}

Our texture orientation estimation relies on Gabor filters since they are known to be accurate (see for instance [12, Section 2.1]). The kernels of the Gabor filters are similar to the $2 \mathrm{D}$ receptive field profiles of the mammalian cortical simple cells and exhibit desirable characteristics of spatial locality and orientation selectivity. For an orientation $\phi$ and a scale (radial frequency) $\omega$, the Gabor wavelets (kernels,filters) are defined by [6]

$$
\psi_{\omega, \phi}(x, y)=\frac{\omega}{\sqrt{2 \pi} c} e^{-\omega^{2}\left(4 a^{2}+b^{2}\right) /\left(8 c^{2}\right)}\left(e^{i a \omega}-e^{-c^{2} / 2}\right)
$$

where $a=x \cos \phi+y \sin \phi, b=-x \sin \phi+y \cos \phi$ and $c=$ 2.2 (octave 1.7 in [6]). We consider 5 scales on a geometric grid and 36 orientations. These parameters are similar to the ones in [12]. Figure 2 shows the real and imaginary parts of the Gabor kernels.

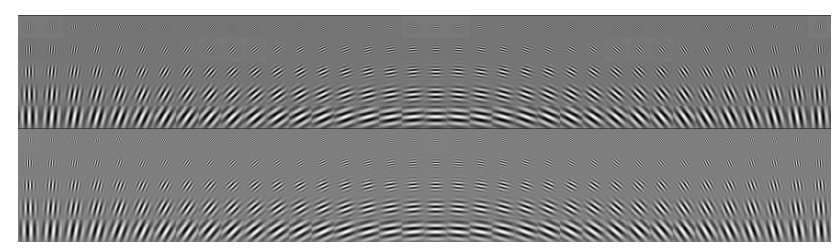

Figure 2. Gabor kernels with 5 scales and 36 orientations: real part kernels (rows 1 to 5) and imaginary part kernels (rows 6 to 10).

Let $I(x, y)$ be the gray level value of an image at $(x, y)$. The convolution of image $I$ and a Gabor kernel of scale $\omega$ and orientation $\phi$ is defined as follows

$$
\mathcal{G}_{\omega, \phi}=I \otimes \psi_{\omega, \phi}
$$

The convolution result $\mathcal{G}_{\omega, \phi}(z)$ at pixel $z=(x, y)$ has two components, a real part and an imaginary part. To best characterize the local texture properties, we compute the square norm of this "complex response" of the Gabor filter for each 36 evenly spaced Gabor filter orientations:

$$
I_{\omega, \phi}(z)=\operatorname{Re}\left(\mathcal{G}_{\omega, \phi}(z)\right)^{2}+\operatorname{Im}\left(\mathcal{G}_{\omega, \phi}(z)\right)^{2} .
$$

The response image for an orientation is then defined as the average of the responses at the different scales (see Fig.3):

$$
R_{\phi}(z)=\text { Average }_{\omega} I_{\omega, \phi}(z) .
$$

The texture orientation $\theta(z)$ is chosen as the filter orientation which gives the maximum average complex response at that location (the average is taken over the 5 scales):

$$
\theta(z)=\operatorname{Argmax}_{\phi} R_{\phi}(z)
$$

The second row of Figure 4 shows the images overlaid with a subsample of the texture orientations estimated using Gabor filters.

From the convolution theorem applied to Eq. (1), we have

$$
\Im\left\{\mathcal{G}_{\omega, \phi}\right\}=\Im\{I\} \Im\left\{\psi_{\omega, \phi}\right\}
$$



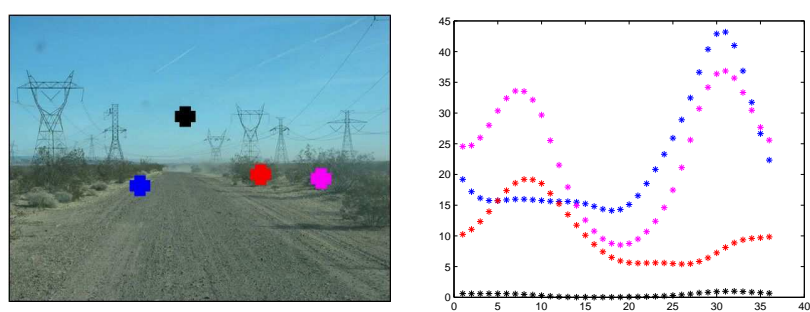

Figure 3. Left: Four points on which the Gabor complex responses are evaluated. Right: The Gabor complex responses for the four points.

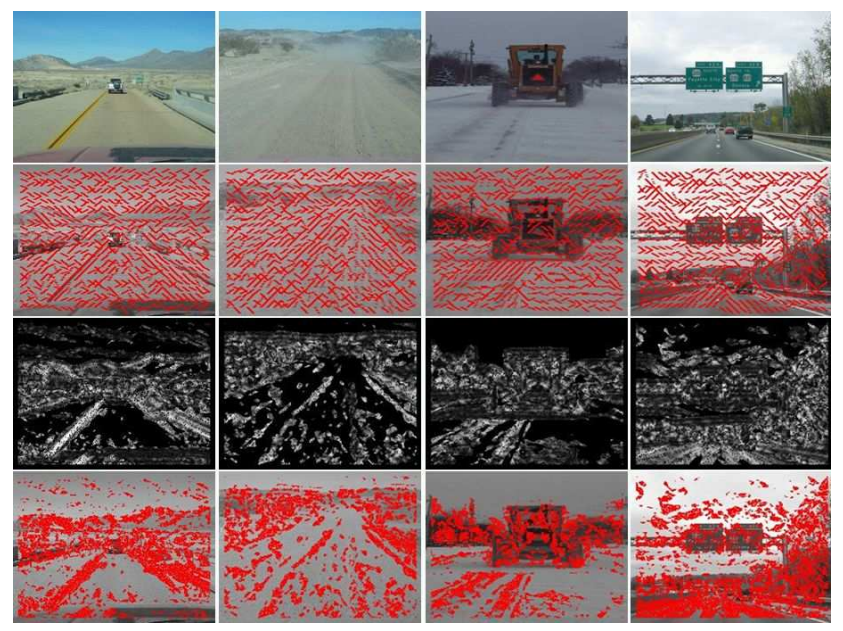

Figure 4. First row: four road sample images. Second row: images overlaid with texture orientations estimated using Gabor fi lters. Third row: examples of the confi dence map for the texture orientation estimation. The brighter the pixel, the higher confi dence the orientation estimation. Fourth row: pixels with confi dence larger than $0.3 \times(\max C o n f-\min \operatorname{Con} f)$, where $\max C o n f$ and $\operatorname{minConf}$ is the largest and smallest confi dence value respectively.

hence

$$
\mathcal{G}_{\omega, \phi}=\Im^{-1}\left\{\Im\{I\} \Im\left\{\psi_{\omega, \phi}\right\}\right\},
$$

where $\Im$ and $\Im^{-1}$ denote the Fourier and inverse Fourier transform, respectively. The use of the fast Fourier transform and of the equatily

$$
\begin{aligned}
& \Im\left\{\psi_{\omega, \phi}\right\}(\xi, \nu) \\
= & \sqrt{8 \pi} \frac{c}{\omega}\left(e^{-c^{2}\left((\alpha-\omega)^{2}+\beta^{2}\right) /\left(2 \omega^{2}\right)}-e^{-c^{2}\left(\alpha^{2}+\omega^{2}+\beta^{2}\right) /\left(2 \omega^{2}\right)}\right),
\end{aligned}
$$

with $\alpha=\xi \cos \phi+\nu \sin \phi$ and $\beta=-\xi \sin \phi+\nu \cos \phi$, allows fast computation of the response image.

Although the above solution for texture orientation estimation has been used by some previous researchers, this estimated texture orientation is not guaranteed to be correct. To provide a confidence level to the texture orientation $\theta(z)$ at pixel $z$, we use how peaky the function $\phi \mapsto R_{\phi}(z)$ is near the optimum angle $\theta(z)$. Let $r_{1}(z)>\cdots>r_{36}(z)$ be the ordered values of the Gabor response for the 36 considered orientations (in particular, $r_{1}(z)=R_{\theta(z)}(z)$ ). The confidence in the orientation $\theta(z)$ is given by

$$
\operatorname{Conf}(z)=1-\frac{\operatorname{Average}\left(r_{5}(z), \ldots, r_{15}(z)\right)}{r_{1}(z)}
$$

In our experiments, we discard the pixels having a confidence score smaller than $0.3\left(\max _{z} \operatorname{Conf}(z)-\right.$ $\left.\min _{z} \operatorname{Conf}(z)\right)$, and consider the remaining pixels as the "voting" pixels. The constant 0.3 can be seen as an arbitrary threshold put on the normalized confidence score.

We did not use directly the magnitude of the response of the Gabor filter, since it leads to worse results than the proposed method. These negative results are mostly due to high magnitudes of the response in parts of the image that are not related to the road and low magnitudes of the Gabor response in the road area, which often happens with unstructured roads and bright sky.

\section{Locally adaptive soft-voting}

After having computed the texture orientation at each pixel of the image, one can make these pixels vote to obtain the vanishing point. Precisely, a pixel $P$ for which the texture orientation is the vector $\bar{O}_{P}$ can vote for all pixels $V$ above $P$ (we consider images in which the road is below the sky) such that the angle $\angle\left((P V), \bar{O}_{P}\right)$ between the direction $(P V)$ and the vector $\bar{O}_{P}$ is below some fixed threshold $\delta$. This "hard-voting" strategy has been used in [12]. In our experiments, we notice that this scheme tends to favor points that are high in the image, leading sometimes to large errors in the estimation of the vanishing point. A typical image for which this defect appears is given in Fig.5.

To deal with this problem, we propose a soft-voting scheme where the voting score received by a vanishing point candidate from a voter is a value taking into account the distance between the vanishing point candidate and the voter. The vanishing point is searched in the top $90 \%$ portion of the whole image, which, to our knowledge, is a realistic assumption for general road images. For each point $V$ of the image, we define a voting region $R_{V}$ as the intersection of the Gabor response image with a half-disk below $V$ centered at $V$ (see Fig.6). The radius of this half-disk is set to be $0.35 \times i m H$, where $i m H$ is the height of the image.

Each pixel $P$ inside $R_{V}$, for which the texture orientation $\bar{O}_{P}$ has been confidently estimated (see end of Section 3), will vote for the candidate vanishing point $V$ all the more as $P$ is close to $V$ and the orientation of its texture coincide with the direction $(P V)$. Specifically, we introduce the ratio $d(P, V)$ equal to the distance between $P$ and $V$ divided by the diagonal length of the image, and let $\gamma=\angle\left((P V), \bar{O}_{P}\right)$ be the angle in degrees between the direction $(P V)$ and the texture orientation at $P$. 


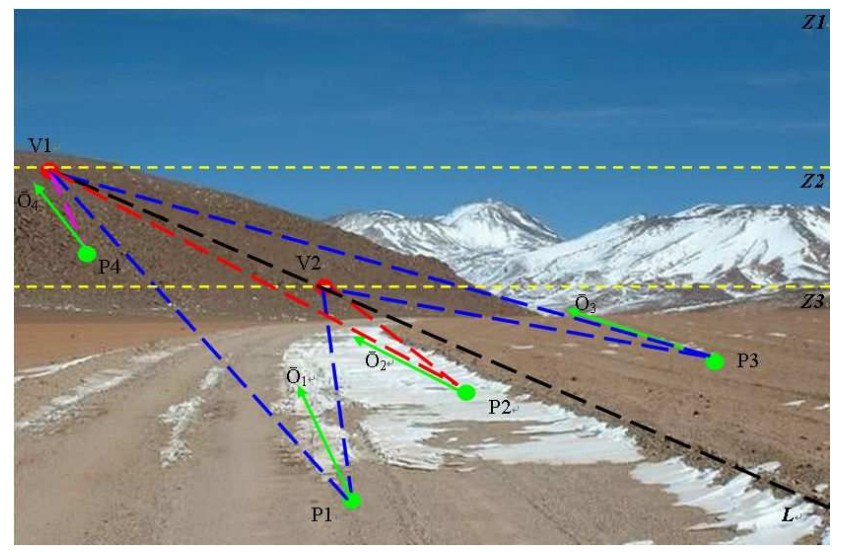

Figure 5. Illustration of the problem in vanishing point estimation by conventional voting strategy. P1, P2, P3 and P4 are four possible voters. $\mathrm{V} 1$ and $\mathrm{V} 2$ are two vanishing point candidates (assuming that $\mathrm{V} 2$ is the true vanishing point). $\bar{O}_{1}, \bar{O}_{2}, \bar{O}_{3}$ and $\bar{O}_{4}$ are respectively the texture orientation vectors of the four voters. The two vanishing point candidates divide the whole image region into three zones, denoted as $Z 1, Z 2$ and $Z 3$. Z1 does not vote for both candidates. Both $Z 2$ and $Z 3$ potentially vote for $\mathrm{V} 1$ while $\mathrm{V} 2$ receives votes only from $Z 3$. Therefore, the higher vanishing point candidates tend to receive more votes than the lower candidates.
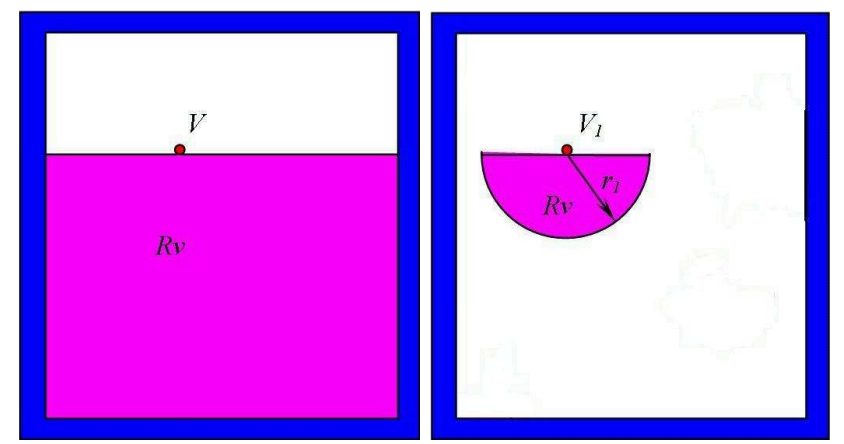

Figure 6. Left: Global $R_{V}$. Right: local $R_{V}$. The blue belt in the images is the border pixels excluded from voting owing to the Gabor convolution kernel size.

$$
\operatorname{Vote}(P, V)= \begin{cases}\frac{1}{1+[\gamma d(P, V)]^{2}} & \text { if } \gamma \leq \frac{5}{1+2 d(P, V)} \\ 0 & \text { otherwise }\end{cases}
$$

Note that the threshold on $\gamma$ also depends on the distance between $P$ and $V$ so that point that are far away (but still within $R_{V}$ ) are taken into account only if the angle $\gamma$ is very small (typically less than $3^{\circ}$ ), while points closer to $V$ will be taken into account up to $\gamma \leq 5^{\circ}$. This allows to limit the influence of points at the bottom of the image and improves the computational efficiency and the accuracy of the results. At the end, the vanishing point is detected as the the candidate that receives the largest voting score.

The advantages of the proposed LASV method over the conventional global hard-voting method lie in three-fold when the true vanishing point does not lie at the very top end of the image. First, the soft-voting strategy suppresses the support to the false vanishing point (i.e., those vanishing point candidates above the true vanishing point) by making the voting score far less than one (unless $\gamma$ is very small). For example, it reduces the support received by $\mathrm{V} 1$ from those voters in $Z 2$ and $Z 3$ in Fig.5. Second, it increases the ratio of the support received by the true vanishing point to that received by the higher false vanishing point, e.g., the support to V2 is larger than that to V1 if P1 votes for both $\mathrm{V} 1$ and V2, while the support to V1 and V2 is equal when using hard-voting method even if P1 votes for both V1 and V2. To discard pixels far away from the vanishing point candidate, or with low confidence in the texture orientation, or with $\gamma$ not small enough results in a significant computational speed-up. Our empirical results show that LASV is more than five times faster than the global hard-voting method [12].

\section{Road segmentation}

The correctly estimated vanishing point provides a strong clue to the localization of the road region. Therefore, we propose a vanishing-point constrained dominant edge detection method to find the two most dominant edges of the road. Based on the two dominant edges, we can roughly segment the road area and update the vanishing point estimated by LASV with the joint point of the two most dominant edges.

The proposed road segmentation strategy is to find the two most dominant edges by initially finding the first one and the other based on the first one. We prefer not to use the color cue in finding these edges because of the following three reasons: Color usually changes with illumination variation. For some road images, there is very subtle or no change in colors between the road and its surrounding areas, e.g., the road covered by snow or desert road. Or for some roads, color changes dramatically in the road area.

For the purposes of easy illustration, the definition of "Orientation Consistency Ratio" (OCR) is given in the top left image of Fig.7: $l$ is a line consisting of a set of discrete oriented points (the orientation of these points denoted by a black arrow in the figure). For each point, if the angle between the point's orientation and the line's direction is smaller than a threshold, this point is viewed to be orientationally consistent with the line. OCR is defined as the ratio between the number of orientationally consistent points and the number of total points on the line. In an image, each point corresponds to a pixel.

We find that the estimated vanishing point coincides with the joint point of a few dominant edges of the road if this vanishing point is a correct estimation, while it usually falls on the extension of one of the most dominant edges if it is a wrong estimation, therefore, we propose to use the initial 

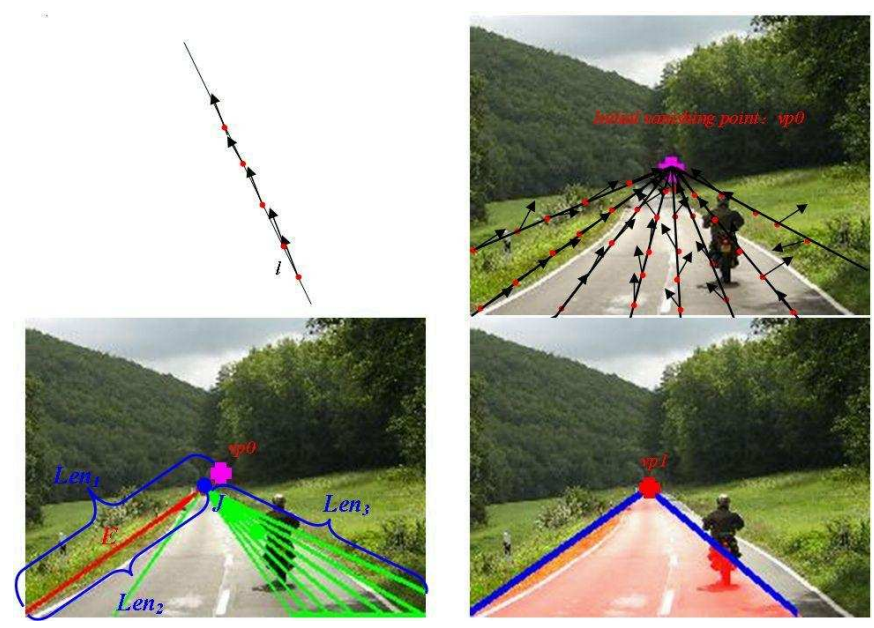

Figure 7. Illustration of detection of the two most dominant edges. Top left: line segments consisting of discrete oriented points. Top right: initially detected vanishing point. Bottom left: detection of the two most dominant edges based on initial vanishing point. Bottom right: the two most dominant edges and updated vanishing point.

vanishing point as a constraint to find the first most dominant edge of the road. The top right image of Fig.7 illustrates this search process, where the first most dominant edge is detected as the one which has the largest OCR from the set of lines going through the initial vanishing point (the angle between two neighboring lines is set to be $5^{\circ}$ ). The red line, $\boldsymbol{E}$, in the bottom left image of Fig.7, is detected as the first most dominant edge and its length is denoted as $L e n_{1}$. To avoid possible false detection caused by short edges, the smallest $L e n_{1}$ is set to be the half image height.

Once the first border of the $\operatorname{road} \boldsymbol{E}$ is found, we will update the initial vanishing point by looking at the points on $\boldsymbol{E}$ having several dominant edges converging to it according to the OCR. For this, through each (regularly) sampled pixel $J$ on $\boldsymbol{E}$, we construct a set of line segments $\left(L_{s}\right)$ such that the angle between any two neighboring of $L_{s}$ is fixed ( $\beta=5^{\circ}$ in our experiments). We also set the angle between $\boldsymbol{E}$ and any one of $L_{s}$ is larger than $20^{\circ}$ (motivated by the assumption that the vanishing angle between the two road borders is larger than $20^{\circ}$ ). We compute the OCR for each line of $L_{s}$, and for each new vanishing point candidate $J$, we consider the sum $S_{J}$ of the top $n$ OCR ( $n=8$ in our experiments). The green line segments in Fig.7 are the $n$ lines starting from $J$ receiving the top $n$ OCR. The vanishing point is then estimate as the point $J$ maximizing $S_{J}$. We try other points along $\boldsymbol{E}$ besides the initial vanishing point since the initial vanishing point estimation may not be accurate (i.e., it is not the joint point of the most dominant edges of the roads). The updated initial vanishing point estimate can be observed in the last three columns of the last five
Table 1. Selection criterion for the second dominant edge

1. Counting the number of dominant edges which deviate to left and right respectively

2. If all deviate to left or right, the two most dominant edges correspond to the two candidates with the largest and smallest deviation angle respectively.

3. Otherwise, find those dominant edges which have different deviation orientation from the first dominant edge 4. Divide these dominant edges into several clusters according to the angle between two neighboring dominant edges, e.g., if the angle is no smaller than $2 \times \beta$, the two neighboring dominant edges belong to different clusters. 5. Find the center of the largest cluster as the deviation angle of the second most dominant edge. If more than one clusters have equal number of dominant edges, the center of these clusters is used.

rows of Fig.10.

From the updated vanishing point and more precisely from the $n$ dominant edges which have voted for it, we deduce the position of the second border of the road as explained in Table 1. The length of the obtained second most dominant edge is denoted $\operatorname{Len}_{3}$ and the length of the first dominant edge is updated to $\operatorname{Len}_{2}$ (see Fig.7). The smallest $L e n_{2}$ and the smallest $L e n_{3}$ are set to be one third of the image height to avoid possible false detections.

\section{Experimental results}

\subsection{Vanishing point estimation}

Vanishing point estimation is tested on 1003 general road images. These road images exhibit large variations in color, texture, illumination and ambient environment. Among them, about 430 images are from the photographs taken on a scouting trip along a possible Grand Challenge route in the Southern California desert and the other part is downloaded from internet by Google Image. Some image samples are shown in Fig.1. All images are normalized to the same size with height of 180 and width of 240 . To assess the algorithm's performance vs. human perception of the vanishing point location, we request 5 persons to manually mark the vanishing point location after they are trained to know the vanishing point concept. A median filter is applied to these human recorded results and the average of the median filter results is regarded as the ground truth position.

For brevity, the soft voting strategy defined in Eq.2 is denoted by "Soft" and the hard voting strategy (by replacing $\frac{1}{1+[\gamma d(P, V)]^{2}}$ with 1 in Eq.2)is denoted as "Hard". The voting strategy based on global voting region (left image of Fig.6) is denoted by "Global" and the one based on local voting region (right image of Fig.6) is denoted by "Local". 
We compare the "Hard" v.s. "Soft" and "Global" v.s. "Local" schemes. We also compare different combination of them with/without introducing the confidence factor. Figure 8 visually gives us the comparison of vanishing point estimation on some sample images. The estimation using the "Hard" and "Soft" voting based on global $R_{V}$ are shown in (a) and (b) respectively, while some results using "Hard" and "Soft" voting based on local $R_{V}$ are shown in (d) and (e) respectively. Figure 8 (c) and (f) shows some samples voted from those image pixels whose confidence score is larger than $0.3 \times(\max C o n f-\min C o n f)$. By comparing (a) with (b) and comparing (d) with (e), it can be observed that "Soft" voting scheme is better than "Hard" voting scheme. By comparing (a) with (d) and comparing (b) with (e), we find that local voting region scheme is more accurate than global voting region one. The examples based on the "Soft" voting from those highly confident texture orientations in the global $R_{V}$ are shown in row (c), and the estimations based on LASV are shown in row (f). Comparing (c) with (a) and (b), and comparing (f) with (d) and (e), we find that it does improve the vanishing point estimation accuracy by introducing the confidence measure.

Figure 9 lists some statistics of the above different combinations. Based on the ground truth positions, we compute the L2 norm distance of the results produced by the above different combinations to the ground truth positions, and put these distances into a 15-bin histogram. If the distance is larger than or equal to 15 , it is put into the 15 th bin of the histogram. The seven histograms are shown in (a) of Fig.9. From Fig.9 (a), we may find that the vanishing point estimation from the pixels with high confidence is much better than the estimation without considering the confidence factor. The threshold, "T", of the confidence is set to be $0.3 \times(\max \operatorname{Con} f-\min \operatorname{Con} f)$. Local votingregion based method produces more accurate estimation than the corresponding global voting-region based method. Based on these histograms, we also compute the percentage of the images whose error distance is smaller than a number. The best results come from the "Soft" voting based on the high-confidence image pixels of the local $R_{V}$ (confidence value is larger than $0.3 \times(\max \operatorname{Con} f-\min C o n f))$ plus updating by the joint point of the two most dominant edges. About $96 \%$ of all images have an error distance no bigger than 10 pixels. The method described in [12] belongs to the "Global"+"Hard" scheme. Based on our experiment, our algorithms perform much better: applying the "Global"+"Hard" scheme to our data, there are 87 images where the true vanishing point is near the bottom of the frame and the error is larger than 50 pixels. In contrast, such a large error occurs in only 33 images for the weakest variant (Global + Soft) of our method. When the error is smaller than 15 pixels, the two methods give similar accuracies. On average, on our test data, our method gives a 9-

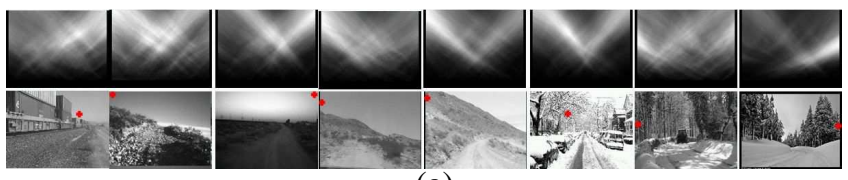

(a)

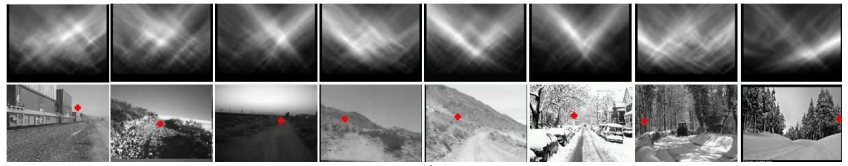

(b)

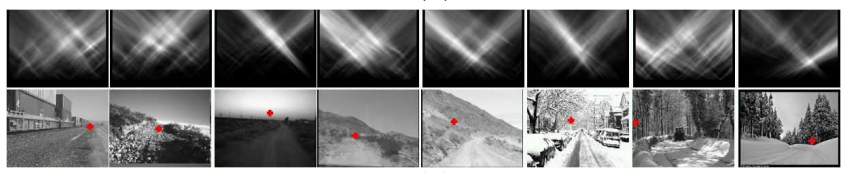

(c)

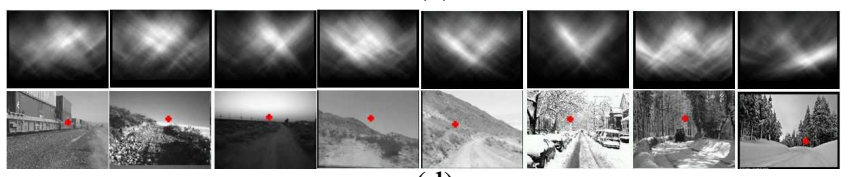

(d)

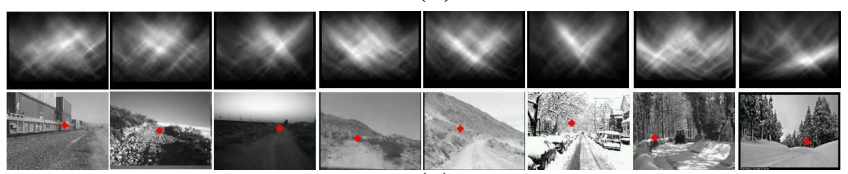

(e)

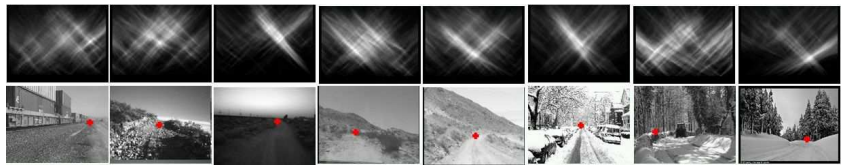

(f)

Figure 8. Comparison of vanishing point estimation based on different combinations.

pixel instead of 14-pixel error for the method in [12]. Note that, for curved road, the vanishing point by our method is located at the joint point of the most immediate straight road borders.

\subsection{Dominant edge detection and road segmenta- tion}

Among the 1003 images, about 300 images are from well paved roads with painted markers. Excluding the 430 desert images, the rest images corresponding to the rural roads have no painted lines although part of them are also well paved. In about $35 \%$ of the well-paved road images with painted lines, the painted line is detected as one of the most dominant edges. For over $90 \%$ of the rural roads, the two road borders are detected as the two most dominant edges. For the desert images, the road can be correctly detected as long as the vanishing point estimation is close to the true position. For curved roads, the detected road region is the most immediately drivable area although part of the road surface cannot be fully encompassed by the two dominant edges. 


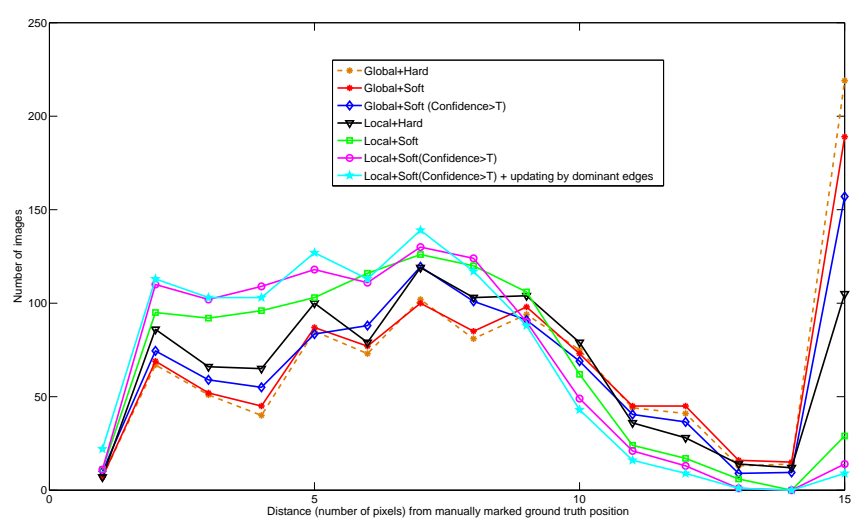

(a)

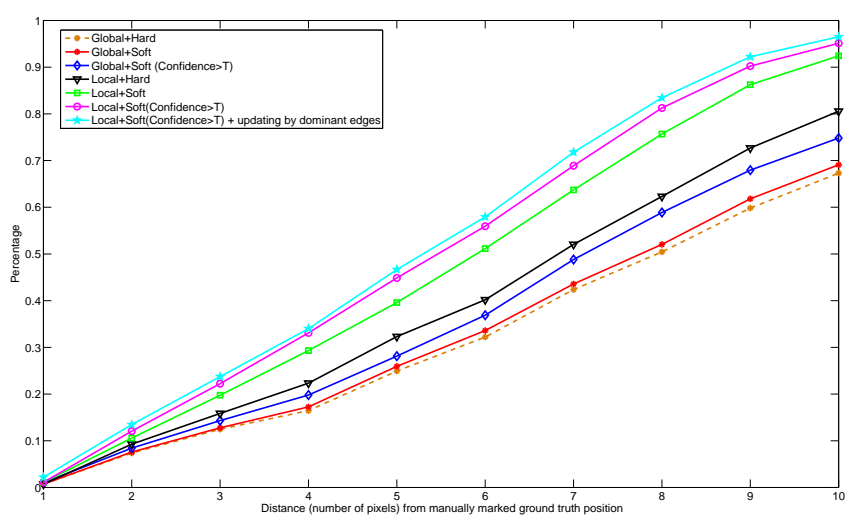

(b)

Figure 9. Comparison of vanishing point estimation accuracy.

Figure 10 (a) corresponds to the desert road images and the bottom five rows comes from the downloaded images. The last three columns of Figure 10 (b) shows us some examples which are wrongly estimated by LASV but corrected by the two dominant edges. The initial vanishing points by LASV are shown in the second row respectively. The detected dominant edge candidates are shown in the third row of them respectively. The two most dominant edges are detected and shown in the fourth row of them respectively. The updated vanishing points by dominant edges are shown in the last row.

\section{Conclusion}

A novel framework for segmenting the general road region from one single image is proposed based on the road vanishing point estimation using a novel scheme, called Locally Adaptive Soft-Voting (LASV) algorithm. Then the estimated vanishing point is used as a constraint to detect two dominant edges for segmenting the road area. To remove the effect caused by noisy pixels, each Gabor texture orien- tation is estimated with a confidence score. In voting, only the pixels of a local voting region whose confidence is high are used, which reduces the computational complexity and improves the accuracy significantly.

\section{Acknowledgements}

This work was supported by a Postdoctoral Fellowship from Evitech and DGA. Thanks to Steven Johnson of the Indiana University for providing the images of the Mojave desert.

\section{References}

[1] Online source: http://www.darpa.mil/grandchallenge.

[2] Y. Alon, A. Ferencz, and A. Shashua. Off-road path following using region classifi cation and geometric projection constraints. CVPR, 2006.

[3] M. Bertozzi and A. Broggi. Gold: A parallel real-time stereo vision system for generic obstacle and lane detection. IEEE Transactions on Image Processing, 1998.

[4] A. Broggi, C. Caraffi, R. I. Fedriga, and P. Grisleri. Obstacle detection with stereo vision for off-road vehicle navigation. Proceedings of IEEE International Workshop on Machine Vision for Intelligent Vehicles, 2005.

[5] W. T. Freeman and E. H. Adelson. The design and use of steerable fi lters. PAMI, 1991.

[6] T. Lee. Image representation using $2 \mathrm{~d}$ gabor wavelets. PAMI, 1996.

[7] A. Lookingbill, J. Rogers, D. Lieb, J. Curry, and S. Thrun. Reverse optical fbw for self-supervised adaptive autonomous robot navigation. IJCV, 2007.

[8] B. Ma, S. Lakshmanan, and A. O. Hero. Simultaneous detection of lane and pavement boundaries using model-based multisensor fusion. IEEE Transactions on Intelligent Transportation Systems, 2000.

[9] R. Manduchi, A. Castano, A. Talukder, and L. Matthies. Obstacle detection and terrain classifi cation for autonomous offroad navigation. Autonomous Robots, 2005.

[10] J. C. McCall and M. M. Trivedi. Video based lane estimation and tracking for driver assistance: Survey, system, and evaluation. IEEE Transactions on Intelligent Transportation Systems, 2006.

[11] M. Nieto and L. Salgado. Real-time vanishing point estimation in road sequences using adaptive steerable fi lter banks. Advanced Concepts for Intelligent Vision Systems, Lecture Notes in Computer Science, 2007.

[12] C. Rasmussen. Grouping dominant orientations for illstructured road following. CVPR, 2004.

[13] C. Rasmussen. Texture-based vanishing point voting for road shape estimation. BMVC, 2004.

[14] J. B. Southhall and C. Taylor. Stochastic road shape estimation. ICCV, 2001.

[15] J. Sparbert, K. Dietmayer, and D. Streller. Lane detection and street type classifi cation using laser range images. IEEE Proceedings in Intelligent transportation Systems, 2001. 


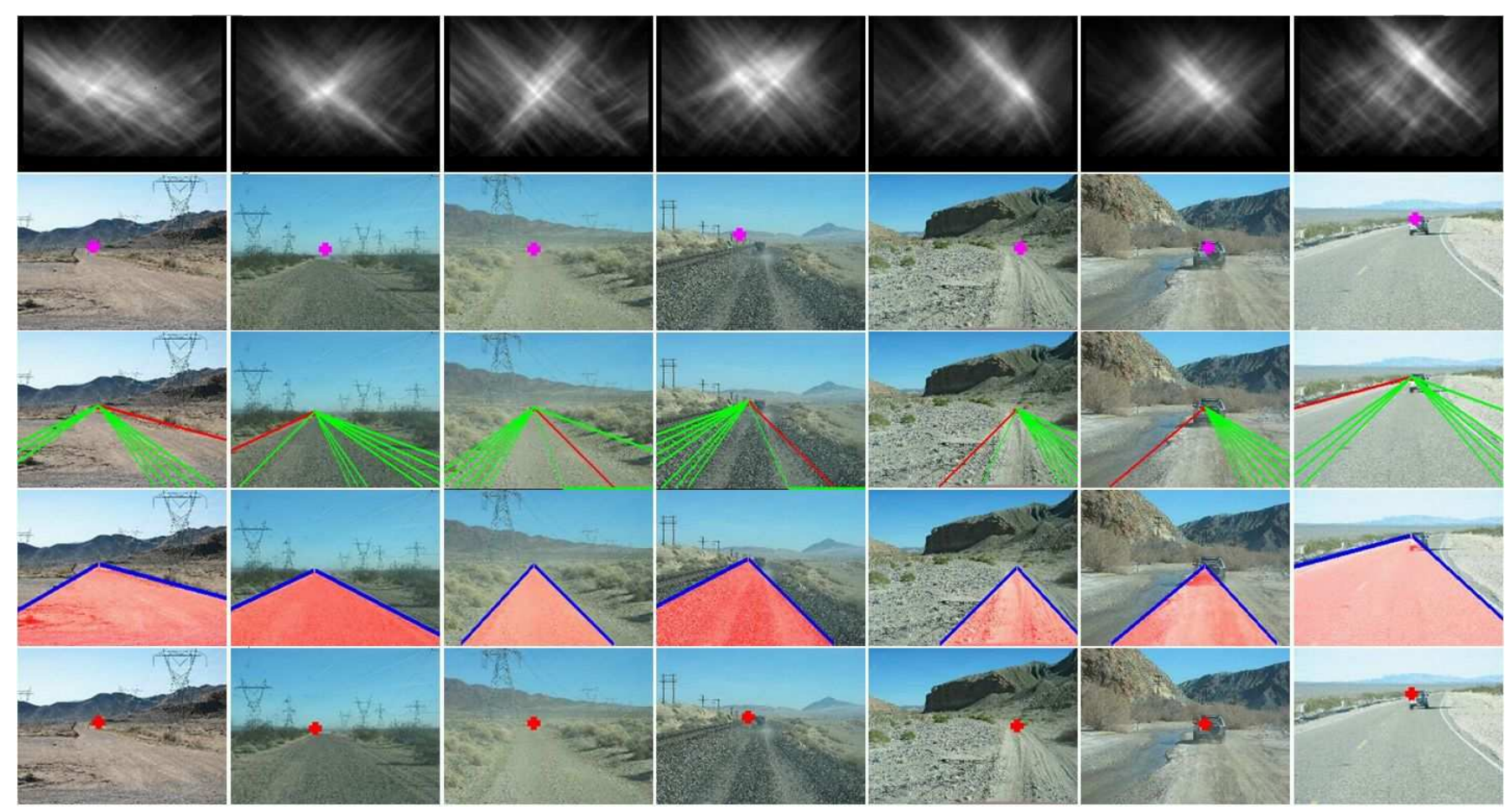

(a)

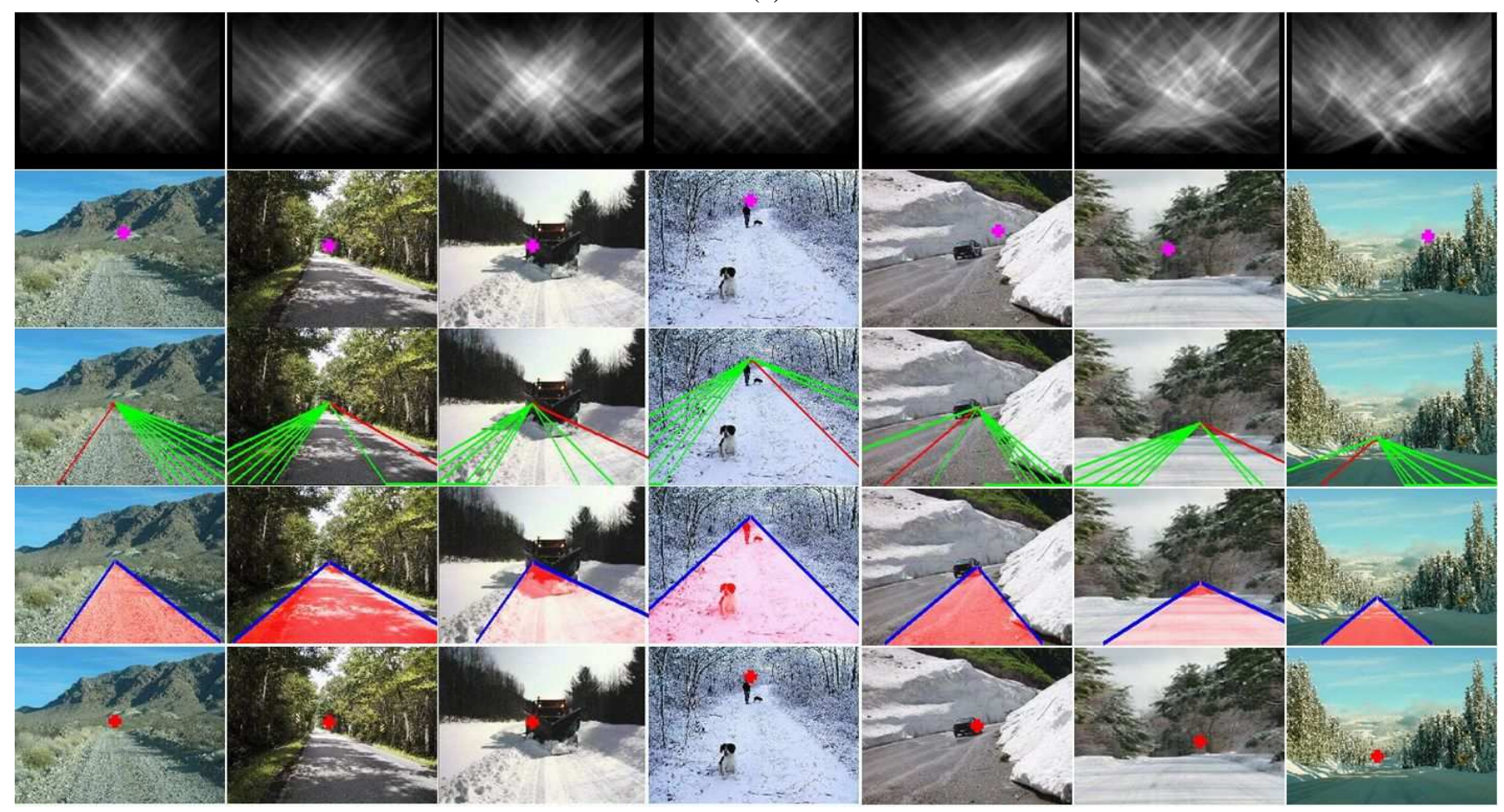

(b)

Figure 10. Vanishing point estimation and dominant edge detection.

[16] S.-J. T. Tsung-Ying Sun and V. Chan. Hsi color model based lane-marking detection. IEEE Intelligent Transportation Systems Conference, 2006.

[17] Y. Wang, E. K. Teoh, and D. Shen. Lane detection and track- ing using b-snake. Image and Vision Computing, 2004.

[18] B. Yu and A. K. Jain. Lane boundary detection using a multiresolution hough transform. ICIP, 1997. 